\title{
PROTEÍNAS MIR-203A-3P E MMP-2 SÃO ALTAMENTE EXPRESSAS EM CÉLULAS TUMORAIS CIRCULANTES DE PACIENTES COM CARCINOMA PANCREÁTICO
}

\author{
MIR-203A-3P AND MMP-2 PROTEINS ARE HIGHLY EXPRESSED IN CIRCULATING TUMOR CELLS FROM PATIENTS \\ WITH PANCREATIC CARCINOMA
}

\author{
José Gabriel Rodríguez TARAZONA ${ }^{\oplus}{ }^{\oplus}$, Emne Ali ABDALLAH ${ }^{1}{ }^{\oplus}$, Bianca de Cássia Troncarelli FLORES ${ }^{1}$, \\ Alexcia Camila BRAUN ${ }^{1 \odot}$, Cláudia Malheiros Coutinho CAMILLO $^{1{ }^{\circ}}$, Fabio Albuquerque MARCHI ${ }^{1}$, \\ Anna Paula Carreta RUANO ${ }^{1}$, Ludmilla Thome Domingos CHINEN ${ }^{\oplus}$
}

RESUMO - RACIONAL: O adenocarcinoma ductal do pâncreas é a quarta causa de morte associada ao câncer mais comum no mundo ocidental. A presença de células tumorais circulantes (CTCS) pode ser considerada um potencial fator prognóstico, visto que essas células representam a progressão tumoral, permitindo o monitoramento da eficácia terapêutica. OBJETIVOS: explorar as características morfológicas, moleculares e fenotípicas das células tumorais circulantes (CTCs) do sangue de pacientes com carcinoma pancreático e correlacionar os achados com a resposta ao tratamento, sobrevida livre de progressão, sobrevida global (SG) e trombose venosa profunda (TVP). MÉTODOS: o sangue periférico $(10 \mathrm{~mL})$ foi analisado antes do início do tratamento e após 60 e 120 dias. As CTCs foram detectadas pelo ISET ${ }^{\oplus}$ e caracterizadas por imunocitoquímica. Para análise de miRNAs, leucócitos periféricos dos mesmos pacientes e indivíduos saudáveis foram coletados em paralelo no início do estudo. A expressão de miRNAs foi avaliada usando TaqMan T Array Human MicroRNA Cards v2.0. RESULTADOS: foram incluídos 9 pacientes. As proteínas MMP2 e TGFB-RI foram altamente expressas $(77,7 \%)$ nas CTCs no início do estudo. No primeiro acompanhamento, MMP2 era predominante (80\%) e no segundo acompanhamento, MMP2 e vimentina eram predominantes (50\%). Microêmbolos tumorais circulantes (MTC) foram encontrados em dois pacientes e ambos apresentavam TVP. O miR203a-3p foi altamente expresso em CTCs. miR-203a-3p está envolvido na estimulação da transição epitelio-mesenquima (TEM) e relacionado a pior SG no câncer pancreático (dados TCGA). CONCLUSÃ̃: Devido ao baixo número de pacientes e curto seguimento, não observamos correlação entre CTCs e resposta ao tratamento. No entanto, houve uma correlação entre MTC e TVP. Além disso, miR-203a$3 p$ foi altamente expresso em CTCs, corroborando os achados de proteínas EMT. Este estudo abre perspectivas sobre a mudança dinâmica no padrão de proteínas expressas ao longo do tratamento e a utilização de miRNAs como novos alvos no carcinoma pancreático.

DESCRITORES: Metaloproteinase 2 da Matriz. Células Neoplásicas Circulantes. Neoplasias Pancreáticas. Trombose.

ABSTRACT - BACKGROUND: Ductal adenocarcinoma of the pancreas is the fourth most common cancerassociated cause of death in the Western world. The presence of circulating tumor cells (CTCs) can be considered a potential prognostic factor, as these cells represent tumor progression, allowing monitoring of therapeutic efficacy. OBJECTIVES: The objectives of this study were to explore the morphological, molecular, and phenotypic characteristics of CTCs from the blood of patients with pancreatic carcinoma and to correlate the findings with response to treatment, progression-free survival, overall survival (OS), and deep vein thrombosis (DVT). METHODS: Peripheral blood $(10 \mathrm{~mL})$ was analyzed before the beginning of treatment after 60 and 120 days. CTCs were detected by using ISET ${ }^{\circledast}$ and characterized by immunocytochemistry. For microRNAs (miRNAs) analysis, peripheral leukocytes from the same patients and healthy individuals (controls) were collected in parallel at baseline. The expression of miRNAs was evaluated (in pool) using TaqMan ${ }^{\circledR}$ Array Human MicroRNA Cards v2.0. RESULTS: Only nine patients were included. The proteins, namely, matrix metalloproteinase-2 (MMP2) and TGF $\beta$-RI, were highly expressed (77.7\%) in CTCs at baseline; at the first follow-up, MMP2 was predominant $(80 \%)$ and, at the second follow-up, MMP2 and vimentin were predominant (50\%). Circulating tumor microemboli (CTMs) were found in two patients and both presented DVT. The miR-203a-3p was highly expressed in CTCs. The miR-203a-3p is involved in the stimulation of epithelial-to-mesenchymal transition (EMT) and is related to worse OS in pancreatic cancer (TCGA data). CONCLUSION: Due to the low number of patients and short follow-up, we did not observe a correlation between CTCs and response to treatment. However, there was a correlation between CTM and DVT and also miR-203a-3p was highly expressed in CTCs, corroborating the findings of EMT proteins. This study opens the perspectives concerning the dynamic change in the pattern of proteins expressed along with treatment and the use of miRNAs as new targets in pancreatic carcinoma.

HEADINGS: Matrix Metalloproteinase 2. Neoplastic Cells, Circulating. Pancreatic Neoplasms. Thrombosis

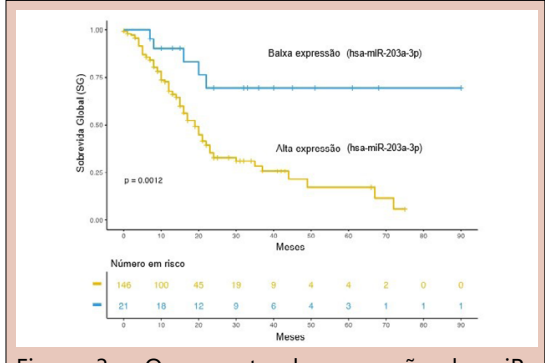

Figura 3 - O aumento da expressão de miR203a-3p está associado a uma sobrevida global pobre em pacientes com câncer de pâncreas com doença em estágio inicial após análise com dados de TCGA.

\begin{tabular}{|l}
\hline Mensagem central \\
\hline A análise dinâmica de proteínas envolvidas na \\
transição epitelial-mesenquimal como também \\
miR-203a-3p em células tumorais circulantes \\
pode ajudar a compreender a biologia do \\
câncer pancreático. A presença de microêmbulos \\
tumorais circulantes se correlacionou com \\
trombose venosa profunda em dois pacientes.
\end{tabular}

\section{Perspectivas}

Este estudo abre perspectivas sobre a mudança dinâmica no padrão de proteínas expressas ao longo do tratamento em células tumorais circulantes e a utilização de miRNAs como novos alvos no carcinoma pancreático.
Correspondência:

Ludmilla Thome Domingos Chinen

Email: ludmilla.chinen@accamargo.org.br

Itdchinen@gmail.com
Fonte de financiamento: Não houve.

Conflito de interesses: Nada a declarar.

Recebido: 04/03/2020

Aceito: 07/07/2020 


\section{INTRODUÇÃO}

$\mathrm{O}$ câncer de pâncreas é atualmente classificado como o $14^{\circ}$ câncer mais comum e a $7^{a}$ principal causa de mortalidade por câncer no mundo. Existe uma tendência geral de maiores taxas de incidência nos países desenvolvidos ${ }^{15}$. Embora não esteja entre os 10 principais cânceres no Brasil, é a oitava principal causa de morte por câncer, devido ao diagnóstico em estágio localmente avançado ou metastático da doença ${ }^{4,20}$. O subtipo histológico mais comum e também mais agressivo de pâncreas câncer é o adenocarcinoma ductal pancreático (ADP), que representa $85-90 \%$ de todas as neoplasias pancreáticas ${ }^{3}$. A ressecção cirúrgica continua como a única modalidade de tratamento com potencial de cura; no entanto, apenas 15-20\% dos pacientes com câncer de pâncreas diagnosticados com a doença são operáveis. No entanto, o nível de recorrências após a cirurgia ainda é alto, tanto local quanto sistêmico. $\mathrm{O}$ tratamento adjuvante é utilizado para melhorar as perspectivas de sobrevida e envolve quimioterapia, radiação e / ou modalidades combinadas, mas ainda existem controvérsias quanto ao tratamento de escolha1.

O CA19-9 tem sido aplicado como "padrão ouro" para monitorar e diagnosticar pacientes com câncer de pâncreas ${ }^{10}$, embora tenha baixo valor preditivo positivo, tornando-o adequado apenas para monitorar a resposta ao tratamento e como marcador de recorrência da doença. A falta de um biomarcador validado e específico para essa doença continua sendo um grande desafio ${ }^{23}$.

As células tumorais circulantes (CTCs) vazam do tumor para a circulação e podem potencialmente invadir tecidos distantes para formar metástases evidentes. Enquanto a maioria das CTCs são células únicas, uma pequena fração viaja como grupos de células². Um microêmbolo tumoral circulante (MTC) é definido como um agrupamento ou grupos de CTCs contendo três ou mais núcleos distintos ${ }^{11}$. MTC parece ter maior potencial metastático do que CTCs individuais em circulação ${ }^{14}$.

Um passo crucial para o intravasamento e sobrevivência na corrente sanguínea é a obtenção de plasticidade e motilidade, que envolve o processo de transição epitélio-mesênquima (TEM) e tanto CTCs quanto MTCs podem passar por esse processo $^{9}$, que pode ser regulado por miRNAs. MicroRNAs (miRNAs) formam redes complexas que regulam a diferenciação, desenvolvimento e homeostase celular. A desregulação de sua função está associada a um número crescente de doenças humanas, principalmente o câncer, sendo, portanto, marcadores de prognóstico ou potenciais alvos de novas terapias contra o câncer ${ }^{1}$. Alguns miRNAs interagem com o conjunto de moléculas críticas envolvidas na engenharia TEM, modulando sua expressão e, consequentemente, sua função ${ }^{12}$.

Assim, este estudo teve como objetivo avaliar a expressão de proteínas relacionadas a TEM e a expressão de miRNAs em CTCs de pacientes com carcinoma pancreático e correlacionar esses achados com dados clínicos, Trombose Venosa Profunda (TVP), Sobrevida livre de progressão (SLP) e sobrevida global (SG).

\section{MÉTODOS}

\section{Declaração Ética}

O protocolo foi aprovado pelo Comitê de Ética em Pesquisa do A.C. Camargo Cancer Center (código 2388/17) de acordo com as orientações do ICH-GCP.

\section{Desenho do Estudo}

Este foi um estudo prospectivo longitudinal unicêntrico realizado por meio da coleta de sangue total de pacientes com carcinoma pancreático, em tratamento com quimioterapia, imunoterapia ou terapia alvo.

Amostras de sangue $(10 \mathrm{~mL})$ foram coletadas antes do início do tratamento, após 60 e 120 dias, com exames de imagem. Como controle negativo, foi utilizado sangue de indivíduos saudáveis.

Este projeto foi submetido ao Comitê de Ética em Pesquisa do A.C. Camargo Cancer Center (código 2388/17). As amostras foram coletadas mediante aceitação e assinatura do Termo de Consentimento Livre e Esclarecido.

Os critérios de inclusão foram: pacientes com diagnóstico histológico de carcinoma pancreático localmente avançado ou metastático; pacientes com mais de 18 anos; pacientes submetidos à primeira linha de tratamento; doença metastática confirmada por avaliação patológica e / ou radiológica; extensão da doença determinada por exame clínico e imagem; doença mensurável pelos critérios RECIST versão 1.1 (Critérios de Avaliação de Resposta em Tumores Sólidos).

Pacientes submetidos a tratamento oncológico prévio foram excluídos.

\section{Isolamento de CTCs e análise de marcadores}

Para separar as CTCs, foi utilizado o método de filtração por tamanho através do dispositivo ISET ${ }^{\circledR}$ (Isolation by SizE of Tumor cells, Rarecells, France). As amostras de sangue periférico do paciente foram coletadas em tubos de EDTA e diluídas para realizar a lise dos glóbulos vermelhos em 1:10 com ISET BufferTM. Após 10 minutos de homogeneização, as amostras foram depositadas em ISET BlockTM, que contém uma membrana de policarbonato com poros circulares de $8 \mu \mathrm{m}$ de diâmetro. O equipamento filtra as amostras por aspiração a vácuo e, em seguida, as membranas são lavadas com Salina Tampada com Fosfato (PBS) $1 \mathrm{X}(\mathrm{pH}: 7,3)$, retiradas do ISET BlockTM e, quando secas, armazenadas a $-20^{\circ} \mathrm{C}$. Dos $10 \mathrm{ml}$ de sangue analisados, $6 \mathrm{ml}$ foram destinados à análise citopatológica e $4 \mathrm{ml}$ à análise molecular (submersão da membrana em RNA posterior e posterior extração de DNA e RNA).

Para caracterizar as CTCs que expressavam as proteínas avaliadas neste estudo, realizamos um ensaio de imunocitoquímica (ICC). Proteínas importantes na TEM foram pesquisadas: antiTGFß-R1 (Cusabio 1: 100, código: CSB-PA061850), anti-mesotelina (Sigma-Aldrich 1:50, código: SAB5500143), anti-Vimentina (Cusabio 1: 100, código: CSB-PA025857LA01HU), anti-MMP2 (Cusabio 1: 100, código: CSB-PA06879A0Rb). Para os controles positivos da reação ICC, usamos linhas de células adicionadas em amostras de sangue saudáveis e filtradas através do ISET ${ }^{\circledR}$. Para o controle do anticorpo anti-TGFß-R1 usamos a linha celular A-549, para o anticorpo anti-mesotelina usamos a linha celular Hela, para o anti-Vimentina usamos a linha celular MCF7 e para o anti-MMP2 usamos a célula U87 linha, de acordo com The Human Protein Atlas (http://www.proteinatlas.org/). Como controle negativo do ICC, usamos a mesma linha celular, omitindo o anticorpo primário, para garantir que a reatividade cruzada fosse excluída. Para confirmar que as CTCs analisadas não eram leucócitos, foi utilizado o anticorpo anti-CD45 (SigmaAldrich 1: 100, código: HPA000440).

O ICC foi realizado com dupla marcação, utilizando o GBI LABS Golden Bridge International Kit (GBI Labs.) com os marcadores desejados. Os pontos da membrana ISET foram cortados e colocados em placas de 24 poços. A recuperação antigênica foi realizada pela adição de $1 \mathrm{ml}$ de (solução de recuperação DakoTM Target $1 \mathrm{X}$ ) de cada poço por aquecimento em um recipiente de micro-ondas com água destilada por aproximadamente 6 minutos com intervalos de resfriamento a cada 1 minuto e 40 segundos. Cada ponto foi hidratado com $160 \mu \mathrm{l}$ de solução salina tamponada com Tris $1 \mathrm{X}$ (TBS 1X, pH: $7,3)$ por 20 minutos. As células foram permeabilizadas com 160 $\mu \mathrm{l}$ de TBS $0,2 \%+$ Triton $\mathrm{X}-100$ durante 5 minutos à temperatura 
ambiente. Após nova lavagem com TBS, as membranas foram incubadas por 15 minutos, no escuro e à temperatura ambiente, com peróxido de hidrogênio, e novamente lavadas com TBS. Em seguida, o anticorpo primário foi aplicado às manchas e incubado durante a noite. As membranas foram lavadas novamente com TBS, incubadas por 30 minutos em Polímero / HRP (Dako, Santa Clara, $C A, E U A)$, lavadas novamente e reveladas pelo cromógeno $D A B$ do mesmo Kit. Em seguida, as membranas foram lavadas com TBS, seguido por 2 horas de incubação com o segundo anticorpo primário e uma incubação de 30 minutos com o polímero AP (GBI Labs). O segundo anticorpo foi revelado por Permanent Red (GBI Labs). previamente diluído de acordo com as instruções do fabricante por 10 minutos. Por fim, o spot foi lavado com água destilada e corado com hematoxilina por 2 minutos, novamente lavado com água destilada e aderido às lâminas para leitura em microscópio de luz em PBS.

As lâminas foram examinadas em microscópio de luz branca, BX61 - Olympus, (Tóquio, Japão) acoplado a uma câmera digital de alta resolução SC100 - Olympus, (Tóquio, Japão). As CTCs foram caracterizadas de acordo com os seguintes critérios: tamanho do núcleo $=16 \mu \mathrm{m}$, irregularidade do contorno nuclear, presença de citoplasma visível, relação núcleo-citoplasma elevada $(>0,8)$. Quando faltava algum dos critérios descritos, as células eram classificadas como atípicas. Os resultados foram dados em número de CTCs por $\mathrm{ml}$ de sangue, de acordo com a análise estatística realizada por Krebs et al. (2012), ${ }^{12}$ contando CTCs em 4 pontos de membrana ou mais. Após análise citopatológica, os valores de CTCs foram somados e a média calculada. Assim, tivemos os cálculos de CTCs em $1 \mathrm{ml}$ de sangue. Além disso, a presença e ausência de MTCs foi incluída na análise.

\section{Análise de expressão de microRNA}

Isolamento de RNA

A extração de RNA foi realizada usando o AllPrep DNA / RNA / miRNA Universal Kit (Qiagen, Hilden, Alemanha). Resumidamente, 4 spots de membrana sem formaldeído foram cortados em pequenos fragmentos (aproximadamente $2 \mathrm{~mm} 2$ ) em microtubos de $1,5 \mathrm{ml}$ e as células foram lisadas usando o tampão RLT Plus (Qiagen) e misturadas por 1 minuto. Posteriormente, as etapas foram seguidas de acordo com as instruções do fabricante.

A concentração de RNA foi medida no espectrofotômetro NanodropT ND-1000 (Thermo Scientific) usando 1,5 $\mu \mathrm{l}$ de amostra e verificando a absorbância a $260 \mathrm{~nm}$.

\section{RT-PCR em tempo real (qPCR)}

Para verificar a qualidade do RNA extraído, um pequeno RNA nuclear U6B (RNU6B) foi amplificado em todas as amostras. A reação de síntese de cDNA específica foi realizada de acordo com os procedimentos recomendados no manual TaqMan ${ }^{\circledR}$ MicroRNA Assays (Applied Biosystems). Apenas as amostras que apresentaram uma curva de amplificação consistente passaram a analisar o perfil de microRNA.

A determinação dos perfis de expressão de miRNA foi realizada usando a metodologia TaqMan T Array Human MicroRNA A Cards v2.0 (contendo 372 miRNAs e 7 controles) de acordo com o kit MegaplexTM Pools for microRNA Expression Analysis (Applied Biosystems). A síntese e pré-amplificação do cDNA foi realizada usando 150ng de RNA de leucócitos de voluntários sem neoplasia (pool de 5 amostras), RNA de leucócitos de pacientes com câncer pancreático (pool de 6 amostras) e RNA de CTCs de pacientes com câncer pancreático câncer (pool de 9 amostras). A amplificação dos microRNAs foi realizada utilizando o $7900 \mathrm{HT}$ Fast Real-Time PCR System (Applied Biosystems).

Os resultados foram analisados pelo software $R Q$ Manager 1.2 (Applied Biosystems). O nível de expressão dos miRNAs foi quantificado em relação à expressão de um miRNA de referência e foi normalizado de acordo com a amostra do calibrador (pool de amostras de leucócitos de voluntários saudáveis). O miRNA utilizado como referência foi o miR-126, selecionado por meio da ferramenta NormFinder (https://moma.dk/normfinder-software), que avalia qual microRNA tem a expressão mais estável em um conjunto de amostras. A quantificação relativa $(\mathrm{Rq})$ foi calculada pelo método do método $\Delta \Delta C T^{13}$.

Os miRNAs foram selecionados de acordo com a maior diferença de expressão entre os grupos CTC e Leucócitos, avaliada por fold-change $=2$ para expressão aumentada $\mathrm{e}=$ -2 para expressão diminuída. Para a construção do boxplot, foi utilizado o pacote ggplot2 disponível para o programa $\mathrm{R}$.

\section{Análise estatística}

Foi realizada uma análise descritiva de cada grupo (aqueles que expressam proteínas relacionadas TEM versus aqueles que não expressam) em relação às variáveis clínicopatológicas. Para avaliar diferenças e associações entre os grupos, o método Qui-quadrado foi usado para variáveis categóricas. Para analisar SLP e SG I, foi utilizado o método de Kaplan-Meier e a diferença entre as curvas calculada por log-rank. Todas as análises estatísticas foram realizadas no programa SPSS for Windows, versão 15 . Os valores de $p$ foram considerados significativos quando $p<0,05$.

Para o cálculo do SG, consideramos o tempo entre a primeira coleta do paciente até o seu óbito e para a SFP, consideramos o tempo entre a primeira coleta do paciente e a progressão objetiva do tumor. A linha de base (primeira amostra) das análises de sobrevivência neste estudo foi realizada a partir da data da primeira coleta de CTCs.

\section{RESULTADOS}

\section{Características clínico-patológicas}

Coletamos amostra de 10 pacientes com adenocarcinoma pancreático antes do procedimento cirúrgico (linha de base) entre 05/2018 e 09/2019. Um paciente foi excluído do estudo porque a análise patológica confirmou que era um tumor neuroendócrino. O primeiro acompanhamento foi realizado dois meses após a coleta da linha de base em 5 pacientes, perdemos 4 pacientes (óbito de um paciente e perda de seguimento de 3 pacientes). O segundo acompanhamento (4 meses após a coleta da linha de base) foi realizado em 8 pacientes.

Dos 9 pacientes incluídos, 4 (44,44\%) eram homens e 5 $(55,55 \%)$ mulheres, a mediana de idade foi de 59,7 anos (42 82). Doadores saudáveis $(n=7), 6$ mulheres e 1 homem, com idade mediana de 48 anos, também foram recrutados como grupo controle para a análise dos experimentos de expressão de microRNA.

Em relação ao grau histológico, 6/9 pacientes apresentavam adenocarcinoma ductal $(66,66 \%), 2 / 9$ pacientes $(22,22 \%)$ adenocarcinoma e em $1 / 9(11,11 \%)$ o tumor foi classificado como carcinoma.

Dos 9 pacientes incluídos, 3 (33,33\%) evoluíram e 6 pacientes permaneceram no mesmo estágio da doença no momento do último acompanhamento. Dos três pacientes que evoluíram, dois tinham doença não metastática e um com doença metastática.

Os pacientes que experimentaram progressão tiveram uma distribuição maior de CTCs na linha de base $(p=0,54)$. As diferenças nos níveis de CTCs ao longo do tratamento são mostradas na Figura 1.

Em relação ao estadiamento patológico dos pacientes incluídos neste estudo, 4 iniciaram o estudo no estágio IV (44,44\%), dois no estágio III (22,22\%), dois no estágio IB (22,22\%) 
e um paciente no estágio IIB $(11,11 \%)$. Em relação às metástases (pM), 5 pacientes $(55,55 \%)$ eram M0 e $4(44,44 \%)$ M1.

Em relação à estratégia terapêutica estabelecida para os pacientes deste estudo, constatou-se nos prontuários que 4 pacientes $(44,44 \%)$ iniciaram tratamento paliativo, três pacientes $(33,33 \%)$ tratamento neoadjuvante e dois pacientes $(22,22 \%)$ tratamento curativo. As demais características clínicas e patológicas dos pacientes estão descritas na Tabela 1.

Dentre os biomarcadores utilizados no estudo, pelos dados do prontuário, observamos que a mediana do nível de CA 19-9 na coleta basal (primeira coleta) foi de $337,9 \mathrm{UI} / \mathrm{ml}$ (3,1 - $41544 \mathrm{UI} / \mathrm{ml})$.

\section{Expressão de Proteína em CTCs}

Avaliamos a expressão de proteínas envolvidas no processo de TEM por imunocitoquímica nas CTCs de 9 pacientes na primeira coleta, 5 na segunda coleta e 8 pacientes na terceira coleta.

Analisamos a expressão proteica das CTCs separadamente, relacionando cada marcador a cada coleção e o que pudemos observar foi a expressão da MMP2 em 77,77\% dos casos da primeira coleção (C1). Na verdade, os pacientes com expressão de MMP2 em CTCs em C1 tiveram uma distribuição mais alta dos níveis de CTC, mas sem significância estatística $(p=0,33)$ (Figuras 1 e 2).

O TGFß-R1 foi expresso em $44,44 \%$ dos casos. Pacientes com expressão de TGFß-R1 nas CTCs em C1 apresentaram maior distribuição dos níveis de CTCs, sem significância estatística ( $p$ $=0,09$ ), assim como a Vimentina.

Não houve diferença estatisticamente significativa no número de casos com presença de proteínas ou correlação com a presença de metástases à distância, ou com progressão da doença em qualquer momento analisado. As expressões das proteínas nas CTCs estão descritas na Tabela 2 e a relação dos marcadores com a evolução clínica na Tabela 3.

\section{Expressão de microRNAs}

A Tabela 4 mostra os 14 melhores miRNAs selecionados no estudo, 13 com menor expressão em CTCs em comparação com os mesmos leucócitos de pacientes e um com maior expressão em CTCs após a mesma comparação com leucócitos de pacientes.

Foram obtidas amostras de 182 amostras de câncer de pâncreas do TCGA (The Cancer Genome Atlas) e selecionadas para a avaliação de miRNAs diferencialmente expressos entre amostras neoplásicas e não neoplásicas. Foram consideradas apenas amostras de pacientes com estágio patológico inicial (I e II), resultando em 50 amostras. Foram considerados miRNAs significativos com $p$-valor $<0,05$ e fold-change $=2 \mathrm{e}=-2$.

Os 93 alvos selecionados foram comparados com os obtidos neste estudo, resultando em 25 candidatos. Observou-se que o miR-203a-3p foi associado a um baixo SO em pacientes com diagnóstico precoce da doença. Pode-se verificar que o aumento da expressão do miR-203a-3p está relacionado ao baixo OS em pacientes com câncer de pâncreas em estágio inicial (Figura 3). Esta análise foi conduzida com o pacote survminer (https://github.com/kassambara/survminer/) de 167 amostras de câncer de pâncreas em estágio inicial que apresentavam informações disponíveis para a curva de sobrevida.

\section{DISCUSSÃO}

Este estudo encontrou CTCs em todos os 9 pacientes com câncer pancreático incluídos. Foram encontradas MMP-2 altamente expressas nessas células, assim como uma correlação entre a presença de MTC e DTV, o que abre perspectivas no acompanhamento de pacientes com câncer de pâncreas, tão propensos a DTV. Como esperávamos, encontramos um

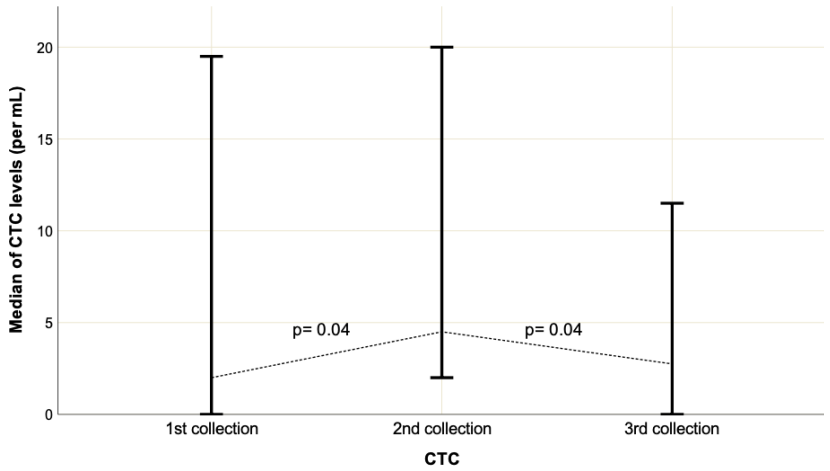

Figura 1 - Mediana de CTCs / $\mathrm{ml}$ nas 3 coletas. Pelo teste de Wilcoxon, observamos que os CTCs de todo o grupo aumentaram com diferença estatisticamente significativa entre a primeira e a segunda coleta $(p=0,04)$, e entre a segunda e a terceira coleta diminuíram com diferença estatisticamente significativa $(p=0,04)$.

Tabela 1 - Características clínicas e patológicas dos pacientes do estudo $(n=9)$. Estadiamento TNM de acordo com AJCC.

\begin{tabular}{|c|c|c|}
\hline Variáveis (n) & $\mathbf{N}^{\circ}$ & $\%$ \\
\hline Número total de pacientes & 9 & 100 \\
\hline \multicolumn{3}{|c|}{ Idade de entrada no estudo, em anos (9) } \\
\hline Média & 57 & \\
\hline Mediana & 59.7 & \\
\hline Variação & $42-82$ & \\
\hline \multicolumn{3}{|l|}{ Gênero (9) } \\
\hline Masculino & 4 & 44,44 \\
\hline Feminino & 5 & 55.55 \\
\hline \multicolumn{3}{|l|}{ Grau histológico (9) } \\
\hline Adenocarcinoma ductal & 6 & 66.66 \\
\hline Adenocarcinoma & 2 & 22.22 \\
\hline Carcinoma & 1 & 11.11 \\
\hline \multicolumn{3}{|l|}{ Diagnóstico do local primário (9) } \\
\hline Neoplasia maligna do pâncreas & 9 & 100 \\
\hline \multicolumn{3}{|l|}{ Metástases no diagnóstico (9) } \\
\hline Sim & 4 & 44.44 \\
\hline Não & 5 & 55.55 \\
\hline \multicolumn{3}{|l|}{ Progressão (9) } \\
\hline Sim & 3 & 33.33 \\
\hline Não & 6 & 66.66 \\
\hline \multicolumn{3}{|l|}{ Trombose (9) } \\
\hline Trombose venosa profunda & 2 & 22.22 \\
\hline Dados não disponíveis & 2 & 22.22 \\
\hline Sem Trombose Venosa Profunda & 5 & 55.55 \\
\hline \multicolumn{3}{|c|}{ Tamanho do tumor (pT) (9) Linha de base } \\
\hline $\mathrm{T} 2$ & 2 & 22.22 \\
\hline T3 & 1 & 11.11 \\
\hline $\mathrm{T} 4$ & 6 & 66.66 \\
\hline \multicolumn{3}{|c|}{$\begin{array}{l}\text { Envolvimento de linfonodos (pN) (9) Linha } \\
\text { de base }\end{array}$} \\
\hline NO & 4 & 44.44 \\
\hline N1 & 5 & 55.55 \\
\hline \multicolumn{3}{|l|}{ Metástases (pM) (9) Linha de Base } \\
\hline MO & 5 & 55.55 \\
\hline M1 & 4 & 44.44 \\
\hline \multicolumn{3}{|l|}{ Estadiamento patológico (9) } \\
\hline IB & 2 & 22.22 \\
\hline III & 2 & 22.22 \\
\hline IIB & 1 & 11.11 \\
\hline IV & 4 & 44.44 \\
\hline \multicolumn{3}{|l|}{ Estratégia Terapêutica (9) } \\
\hline Neoadjuvante & 3 & 33.33 \\
\hline Paliativo & 4 & 44.44 \\
\hline Curativo & 2 & 22.22 \\
\hline
\end{tabular}


microRNA relacionado à TEM altamente expresso nas CTCS desses pacientes, miR-203a-3p. Assim, apesar do pouco material genético, devido ao baixo número de CTCs isoladas de pacientes, obtivemos informações importantes, que podem servir como novos alvos terapêuticos. Acreditamos que o modelo do pool amostral de nosso experimento permite dimensionar alvos que em apenas um paciente não puderam ser encontrados, devido à diversidade e heterogeneidade dos CTCs.

As metaloproteinases de matriz (MMP) são uma família de enzimas proteolíticas que degradam vários componentes da matriz extracelular, incluindo aqueles na membrana basal dos vasos. Estão envolvidos na invasão tumoral, neoangiogênese e metástase (22). A MMP2 (gelatinase A) decompõe o colágeno

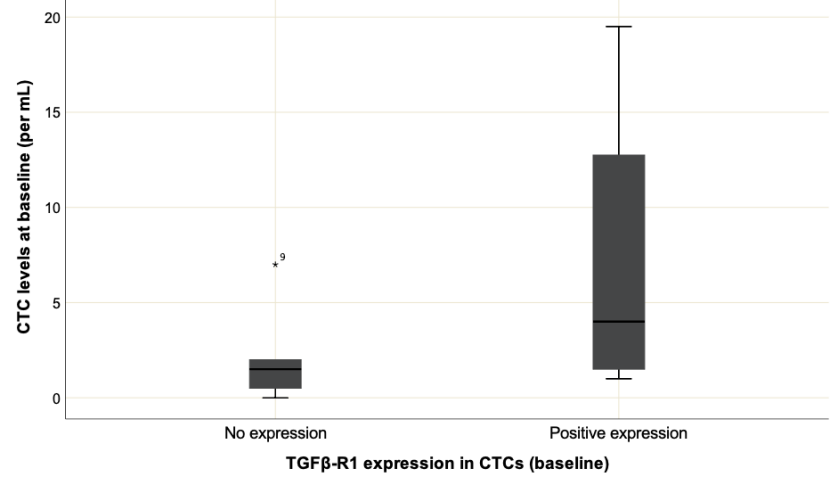

Figura 2 - Distribuição das CTCs na expressão da proteína TGFßR1 na primeira coleta. Não tem significância estatística $(p=0,09)$. A distribuição quantitativa das variáveis foi calculada usando o teste $U$ de Mann-Whitney. tipo IV, a gelatina, a elastina e os proteoglicanos. É regulado em diversos tipos de câncer, como glioblastomas, melanomas, câncer de mama e câncer de cólon, promovendo invasão e metástase. Foi demonstrado que a inibição da MMP2 causa radiossensibilização, diminuição do crescimento tumoral e da invasividade, identificando esta enzima como um alvo interessante para o desenvolvimento de abordagens diagnósticas e terapêuticas ${ }^{17}$.

Murray et al. ${ }^{16}$ estudaram a expressão de MMP2 em células circulantes da próstata (CCPs), células tumorais disseminadas (CTDs) e micrometástases (mM) na medula óssea de homens com câncer de próstata por imunocitoquímica. Em 185 pacientes com câncer não metastático, CCPs foram detectados em $62,7 \%$, CTDs em $62,2 \%$ e mM em 71,4\%. Em 30 pacientes com câncer metastático, $100 \%$ dos CCPs, CTDs e mM foram detectados. Em todos os CCPs, CDTs e mM, a expressão de MMP2 foi associada positivamente a um aumento no escore de Gleason. A expressão de MMP2 em CCPs e CTDs mostrou concordância. Nossos achados, embora em câncer de pâncreas, demonstraram que a expressão de MMP2 foi a maior de todas as proteínas do estudo em todas as coleções, sendo a maior expressão encontrada na segunda coleção, em $80 \%$ dos pacientes.

Poruketal. ${ }^{18}$ estudaram o sangue periférico e portal adequado de 50 pacientes com CDP antes da ressecção cirúrgica e filtradas com ISET. As CTCs foram identificadas por imunofluorescência para positividade de citoqueratina e vimentina. A citoqueratina foi expressa em $78 \%$ dos pacientes e a vimentina em $67 \%$. A detecção de CTCs que expressam vimentina e citoqueratina foi preditiva de recorrência $(p=0,01)$. Em nosso estudo, a vimentina não foi altamente expressa na primeira coleção, embora sua expressão na segunda coleção tenha aumentado, o que pode levar à hipótese de ser um mecanismo de resistência à quimioterapia após o início da quimioterapia.

Tabela 2 - Dados dos biomarcadores do estudo, taxa de detecção de CTC, marcadores em CTCs em todos os momentos do estudo $(n=9)$.

\begin{tabular}{|c|c|c|}
\hline Variáveis (n) & $\mathbf{N}^{\circ}$ & $\%$ \\
\hline \multicolumn{3}{|l|}{ Biomarcadores } \\
\hline Nível médio de CA 19-9 (UI / mL) Linha de base & $337.9(3.1-41544)$ & \\
\hline Nível médio de linha de base CA 19-9 (UI / mL) & $7841.7(3.1-41544)$ & \\
\hline \multicolumn{3}{|l|}{$N^{\circ}$ mediana de CTCs / mL } \\
\hline $1^{\text {a }}$ coleta (avaliada em 09/9) & $2(0-19.5)$ & \\
\hline $2^{\mathrm{a}}$ coleta (avaliada em 09/05) & $4.5(2-20)$ & \\
\hline $3^{a}$ coleta (avaliada em 9/9) & $2.75(0-11.5)$ & \\
\hline \multicolumn{3}{|l|}{ Pacientes com presença de CTCs } \\
\hline $1^{\text {a }}$ coleta (avaliada em 09/9) & 8 & 88.88 \\
\hline $2^{a}$ coleta (avaliada em 09/05) & 5 & 55.55 \\
\hline $3^{a}$ coleta (avaliada em 9/9) & 7 & 77.77 \\
\hline Coleções totais (22) & 20 & 90.90 \\
\hline \multicolumn{3}{|c|}{ Pacientes com células tumorais circulantes positivas para MMP2 } \\
\hline $1^{\text {a }}$ coleta (avaliada em 9) & 7 & 77.77 \\
\hline $2^{a}$ coleta (avaliada 5 ) & 4 & 80 \\
\hline $3^{a}$ coleta (avaliada em 8 ) & 4 & 50 \\
\hline \multicolumn{3}{|c|}{ Pacientes com células tumorais circulantes positivas para VIMENTINA } \\
\hline $1^{\text {a }}$ coleta (avaliada em 9) & 3 & 33.33 \\
\hline $2^{a}$ coleção (avaliada em 5) & 3 & 60 \\
\hline $3^{a}$ coleção (avaliada em 8) & 2 & 25 \\
\hline \multicolumn{3}{|c|}{ Pacientes com células tumorais circulantes positivas para TGFB-R1 } \\
\hline $1^{\text {a }}$ coleta (avaliada em 9 ) & 4 & 44.44 \\
\hline $2^{a}$ coleta (avaliada 5 ) & 3 & 60 \\
\hline $3^{a}$ coleta (avaliada em 8 ) & 1 & 12.5 \\
\hline \multicolumn{3}{|l|}{ Pacientes com microêmbolos tumorais circulantes } \\
\hline $1^{a}$ coleta (avaliada em 9 ) & 1 & 11.11 \\
\hline $2^{\mathrm{a}}$ coleta (avaliada 5 ) & 0 & 0 \\
\hline $3^{a}$ coleta (avaliada em 8 ) & 1 & 12.5 \\
\hline
\end{tabular}




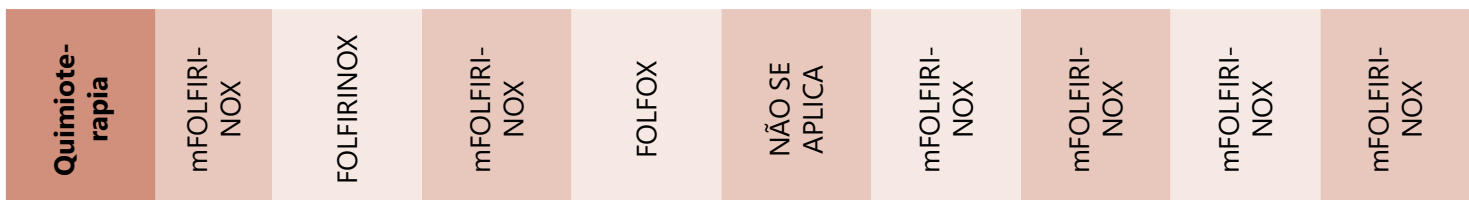

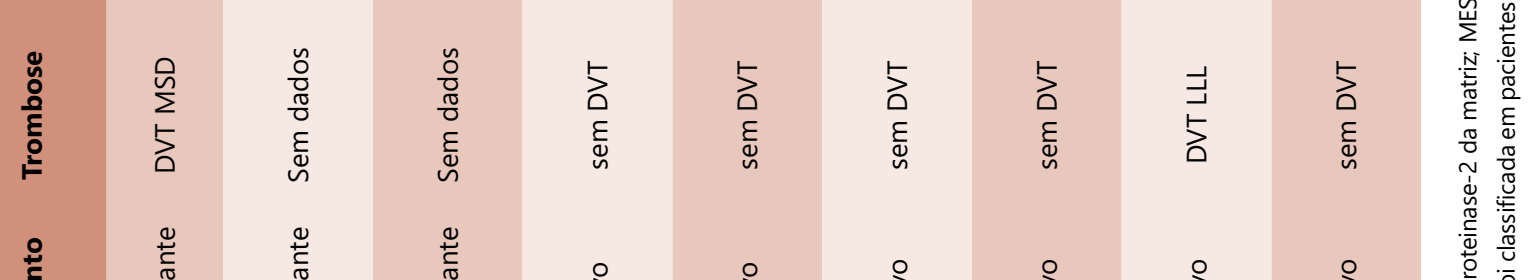

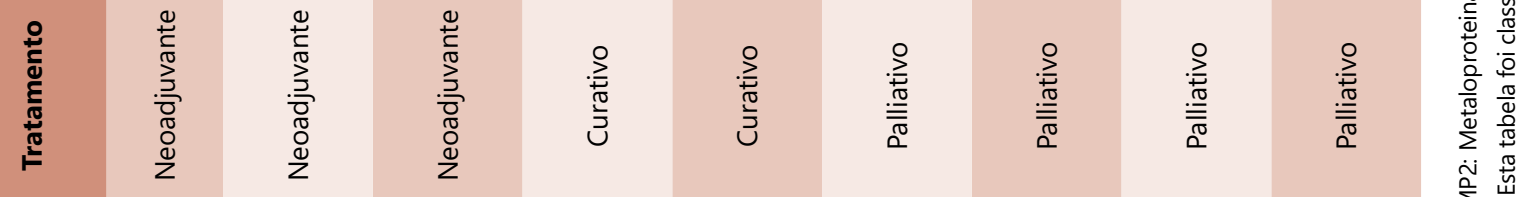

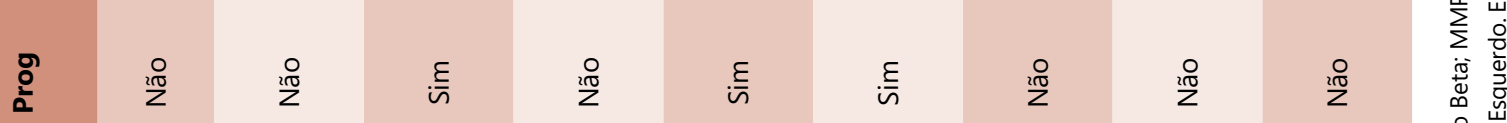

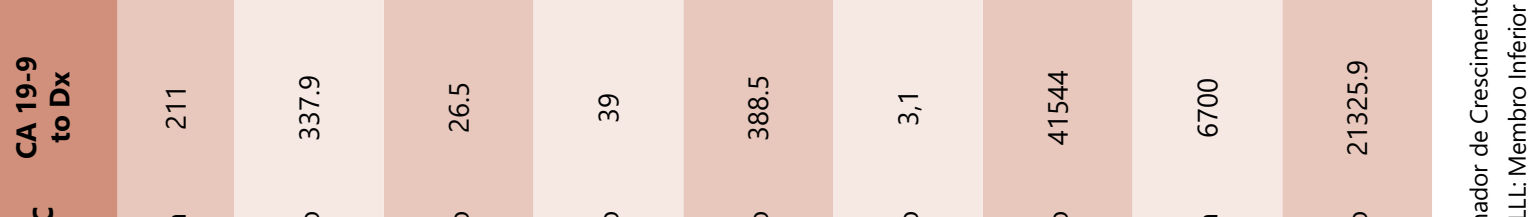

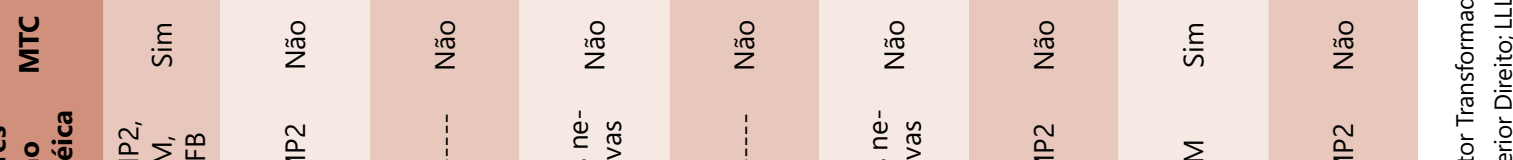

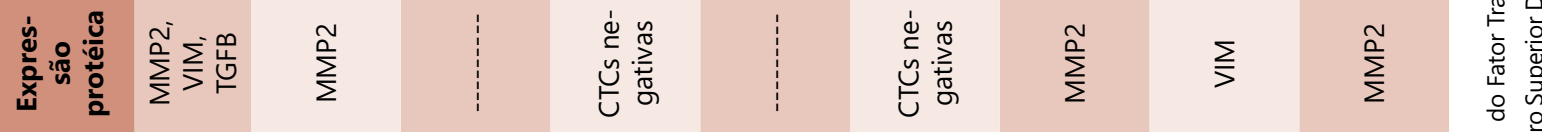

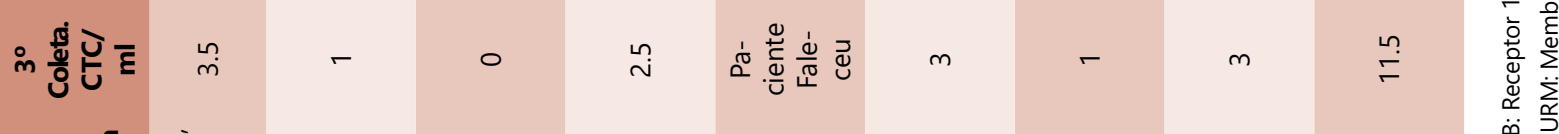

$\frac{\dot{0}}{\frac{3}{2}}$

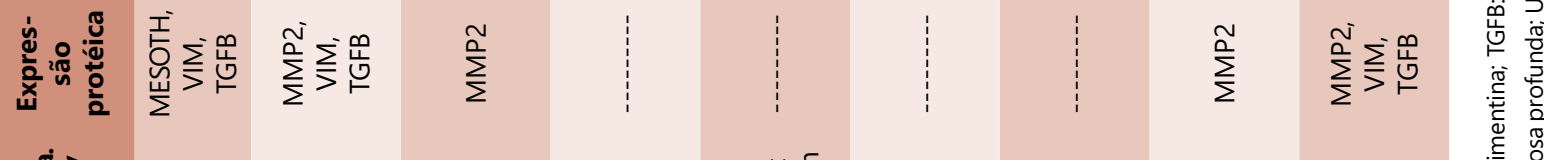

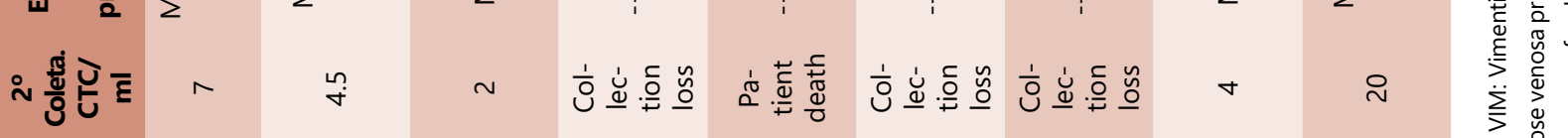

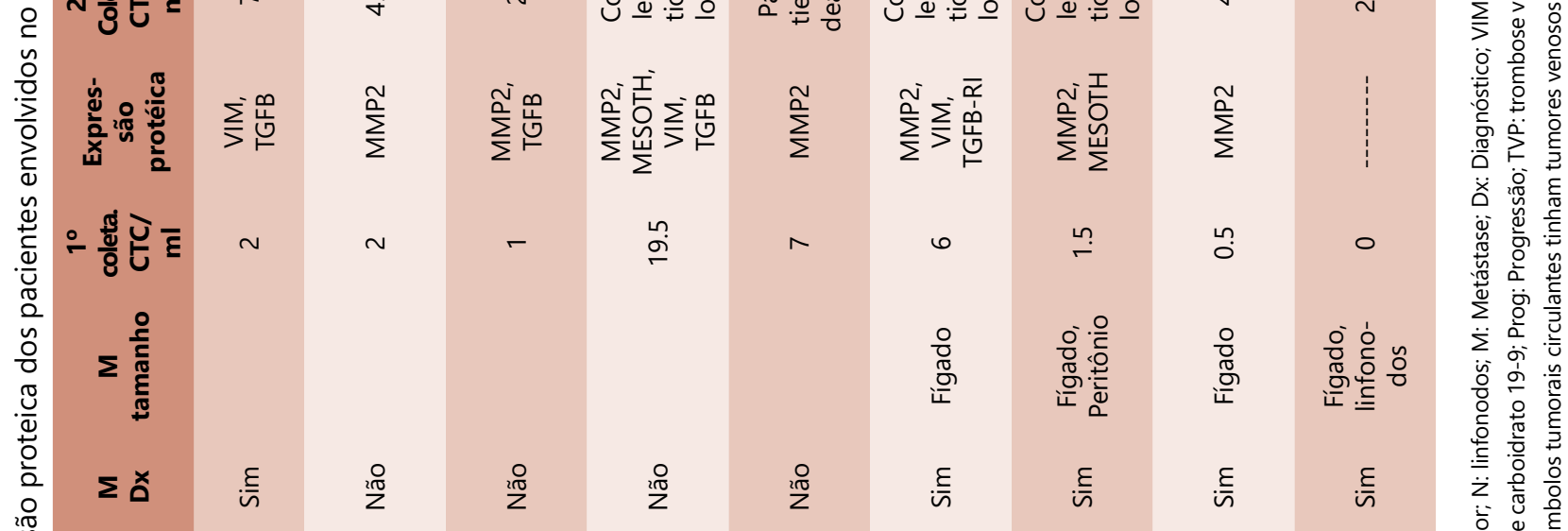

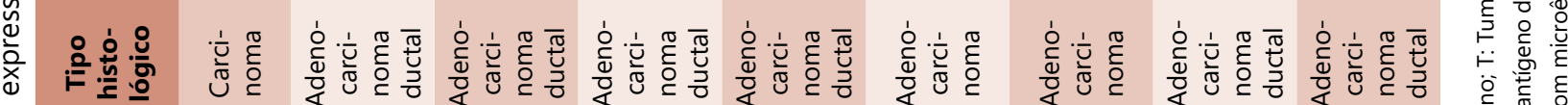

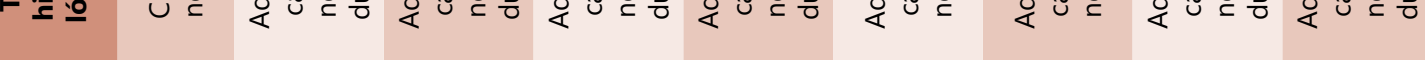

릉

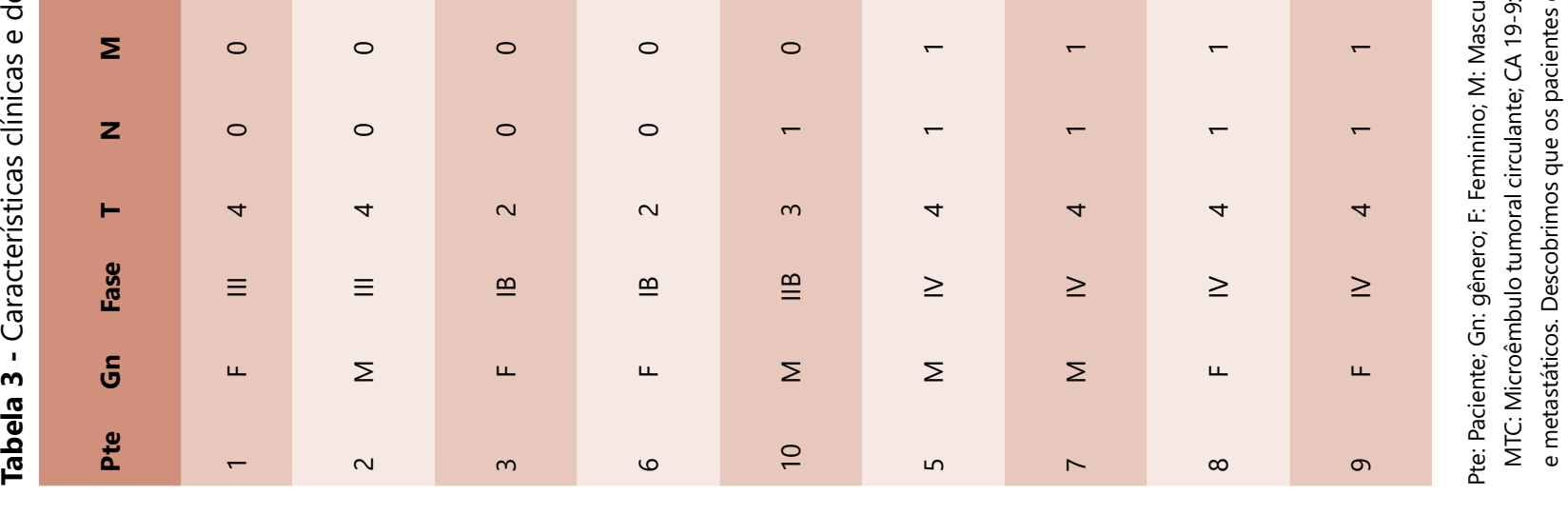


Tabela 4 - Comparação entre miRNAs diferencialmente expressos em CTCs e em tumores primários obtidos em TCGA. 14 miRNAs concordaram com a expressão entre CTCs e tumor (13 downexpressed e 1 overexpressed).

\begin{tabular}{|c|c|c|c|c|}
\hline miRNAs (CTCs) & FC & Pval & Study & Status \\
\hline miR-132-5p* & $-1,72$ & $1,78 \mathrm{E}-02$ & $-38,64$ & baixo \\
\hline miR-143-5p* & $-1,80$ & 4,44E-02 & $-26,28$ & baixo \\
\hline miR-197-3p* & $-1,88$ & $7,41 \mathrm{E}-04$ & $-21,87$ & baixo \\
\hline miR-142-3p* & $-5,26$ & $5,08 \mathrm{E}-05$ & $-8,66$ & baixo \\
\hline miR-223-3p* & $-3,93$ & $2,90 \mathrm{E}-03$ & $-7,29$ & baixo \\
\hline miR-223-5p* & $-2,70$ & $3,06 \mathrm{E}-03$ & $-7,29$ & baixo \\
\hline miR-142-5p* & $-3,48$ & $1,50 \mathrm{E}-03$ & $-6,93$ & baixo \\
\hline miR-145-5p* & $-2,23$ & $3,56 \mathrm{E}-03$ & $-6,67$ & baixo \\
\hline miR-145-3p* & $-1,75$ & $1,81 \mathrm{E}-02$ & $-6,67$ & baixo \\
\hline miR-22-5p* & $-2,94$ & $9,06 \mathrm{E}-06$ & $-6,06$ & baixo \\
\hline miR-345-5p* & $-2,11$ & 2,62E-03 & $-4,03$ & baixo \\
\hline miR-340-3p* & $-2,22$ & $2,18 \mathrm{E}-04$ & $-3,94$ & baixo \\
\hline miR-340-5p* & $-1,74$ & $3,46 \mathrm{E}-02$ & $-3,94$ & baixo \\
\hline$m i R-150-5 p$ & $-3,73$ & $2,26 \mathrm{E}-02$ & $-2,86$ & baixo \\
\hline$m i R-150-3 p$ & $-2,56$ & 2,19E-03 & $-2,86$ & baixo \\
\hline miR-191-5p & $-2,46$ & $1,28 \mathrm{E}-05$ & $-2,72$ & baixo \\
\hline miR-342-3p & $-2,80$ & $3,64 \mathrm{E}-04$ & $-2,66$ & baixo \\
\hline$m i R-18 a-5 p$ & $-2,07$ & $3,22 \mathrm{E}-03$ & $-2,63$ & baixo \\
\hline$m i R-18 a-3 p$ & $-1,46$ & $1,47 \mathrm{E}-02$ & $-2,63$ & baixo \\
\hline miR-590-5p & $-1,88$ & $3,81 \mathrm{E}-03$ & $-2,54$ & baixo \\
\hline miR-362-3p & $-1,83$ & 4,77E-03 & $-2,26$ & baixo \\
\hline$m i R-324-5 p$ & $-1,92$ & $9,09 E-03$ & $-2,11$ & baixo \\
\hline miR-28-3p & $-1,59$ & $4,86 \mathrm{E}-02$ & $-2,05$ & baixo \\
\hline$m i R-28-5 p$ & $-1,43$ & $2,62 E-02$ & $-2,05$ & baixo \\
\hline miR-203a-3p* & 3,90 & $3,53 \mathrm{E}-02$ & 35,80 & alto \\
\hline
\end{tabular}

T: Tumor; NT: Tecido Normal; FC: Fold-Change; Pval: valor P. O asterisco (*) indica os miRNAs que apresentaram o perfil de expressão com a mesma direção dos miRNAs identificados neste estudo.

Em estudo anterior de nosso grupo, Gasparini-Junior et al. ${ }^{8}$ estudaram a expressão de TGFß-RI em pacientes com adenocarcinoma ductal pancreático e 16 pacientes foram testados para TGFß-RI, 4 apresentando marcação positiva (25\% dos pacientes). Não houve diferença estatisticamente significativa no SLP entre os pacientes que apresentaram marcação positiva para TGFß-RI em comparação com aqueles que não o fizeram. No entanto, quando TGFB-RI e MMP-2 foram avaliados juntos, aqueles pacientes que apresentaram um ou ambos (marcadores mesenquimais) em CTCs, progrediram mais rapidamente do que aqueles sem quaisquer marcadores mesenquimais $(2,84 \mathrm{vs}$. 5,43 meses), embora sem significância estatística diferença ( $p=$ $0,14)$. Nosso estudo permitiu ver a cinética desta proteína nas três coletas, percebendo um aumento no segundo momento, apresentando-se em $60 \%$ dos pacientes e com uma queda importante da expressão no terceiro momento das coletas, o que pode expor que o fenótipo mesenquimal é mais exibido após o início da quimioterapia, podendo, talvez, representar um mecanismo de resistência tumoral.

A vimentina, um dos principais constituintes da família das proteínas do filamento intermediário, é onipresente nas células mesenquimais normais e é conhecida por manter a integridade celular e fornecer resistência contra o estresse. $\mathrm{O}$ aumento da expressão de vimentina foi relatado em vários
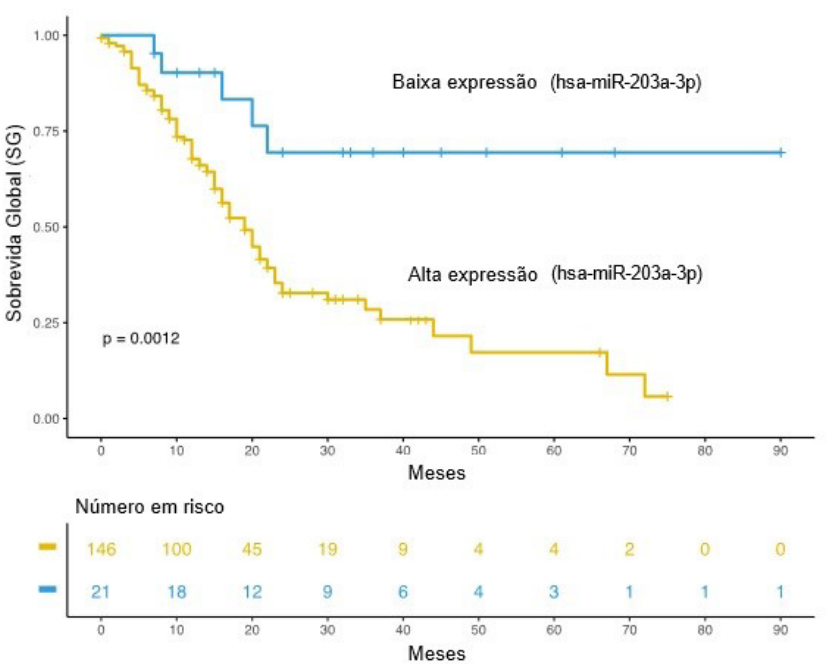

Figura 3 - O aumento da expressão de miR-203a-3p está associado a uma sobrevida global pobre em pacientes com câncer de pâncreas com doença em estágio inicial após análise com dados de TCGA.

cânceres epiteliais (próstata, mama, melanoma, pulmão, tumores gastrointestinais e do CSN), correlacionando-se com aumento do crescimento tumoral, invasão e mau prognóstico. No entanto, o papel da vimentina na progressão do câncer permanece obscuro, apesar de ser amplamente utilizado como um marcador para $\mathrm{TEM}^{19}$. A maior expressão de vimentina nas células do câncer pancreático pode implicar em um maior estado de malignidade ${ }^{24}$.

Um estudo recente analisou se a vimentina na superfície celular poderia ser um biomarcador para isolar CTCs em adenocarcinoma ductal pancreático (ADP) e observou que essa proteína era altamente expressa em células tumorais pancreáticas com fenótipo mesenquimal. CTCs positivos para vimentina (CTCvim) foram detectados em $76 / 100$ pacientes com $A D P$, usando um ensaio microfluídico. Contagens de CTCvim correlacionadas com mudanças na carga tumoral para pacientes submetidos à ressecção. As contagens de CTC significativamente reduzidas foram observadas após a quimioterapia em indivíduos que responderam ao tratamento. Contagens mais altas de CTC no período pré-operatório se correlacionaram com menor sobrevida livre de recorrência. Juntos, vimentina e CTCs podem ser biomarcadores confiáveis no ADP ${ }^{21}$.

Corroborando nossos achados com miR-203a-3p, ${ }^{6}$ realizaram pequenas sequências de RNA de uma linha celular epitelial da mama (D492) e seu derivado mesenquimal (D492M) cultivado em um microambiente tridimensional. Entre os miRNAs mais regulados em D492M estava o miR-203a, um miRNA que desempenha um papel importante na diferenciação epitelial. O aumento da expressão de miR-203a foi observado em D492. Quando miR-203a foi superexpresso em D492M, uma reversão parcial em direção ao fenótipo epitelial foi observada. A análise da expressão gênica de D492M e D492MmiR-203a revelou peroxidasina, envolvida na produção de colágeno IV, como o gene mais significativamente regulado em D492MmiR203a. A superexpressão de miR-203a em D492M induziu TEM parcial e reduziu a expressão de peroxidasina. Além disso, os autores demonstraram que miR-203a é um novo repressor de peroxidasina. O eixo MiR-203-peroxidasina pode ser um importante regulador de TEM/TME e remodelação da membrana basal. Nas CTCs, este microRNA pode estar envolvido nesses mecanismos, estudos adicionais são necessários para melhor compreender esta via e seu papel nas CTCs de pacientes com câncer de pâncreas. 
Chen et al. ${ }^{7}$ estudos tiveram como objetivo fornecer um novo alvo terapêutico (LINC00342) para terapia CPNPC. A expressão do alvo foi detectada por PCR em tempo real (qRTPCR). A migração e invasão celular foram medidas por ensaio transwell. As ferramentas DIANA do software online foram usadas para prever a conexão do LINC00342 e miR-203a-3p pela luciferase. A expressão de LINC00342 foi aumentada em tecidos e células CPNPC em comparação com tecidos e células normais. A eliminação de LINC00342 suprimiu a proliferação celular, formação de colônias, migração e invasão. O estudo sugeriu que LINC00342 contribui para o crescimento e metástase de células CPNPC por meio do direcionamento competitivo de miR-203a-3p.

\section{CONCLUSÃO}

Apesar da coorte super-restrita, nossos dados abrem perspectivas para novas formas de avaliação de CTCs, não apenas analisando-as sob o aspecto citopatológico, mas também em nível de microRNAs. Esta informação associada, em um estudo bem desenhado, pode ser útil no manejo clínico e terapêutico de pacientes com adenocarcinoma pancreático.

\section{REFERÊNCIAS}

1. Abba M, Patil N, Leupold J, Allgayer H. MicroRNA Regulation of Epithelial to Mesenchymal Transition. J Clin Med. 2016;5(1):8. doi: 10.3390/jcm5010008.

2. Aceto N, Toner M, Maheswaran S, Haber DA. En Route to Metastasis: Circulating Tumor Cell Clusters and Epithelial-toMesenchymal Transition. Trends in Cancer. 2015;1(1):44-52. doi: 10.1016/j.trecan.2015.07.006.

3. Åkerberg $D$, Ansari $D$, Andersson R, Tingstedt B. The Effects of Surgical Exploration on Survival of Unresectable Pancreatic Carcinoma: A Retrospective Case-Control Study. J Biomed Sci Eng. 2017;10:1-9. https://doi.org/10.4236/jbise.2017.101001.

4. Ardengh JC, Brunaldi VO, Brunaldi MO, Gaspar AF, Lopes-Júnior JR, Sankarankutty AK, Kemp R, Santos JSD. Is the new procore $20 \mathrm{~g}$ double forward-bevel needle capable to obtain better histological samples by endoscopic ultrasound for diagnosing solid pancreatic lesions? Arq Bras Cir Dig. 2021;33(4):e1554. doi: 10.1590/0102$672020200004 \mathrm{e} 1554$.

5. Blaszak M, El-Masri M, Hirmiz K, Mathews J, Omar A, Elfiki T, Gupta R, Hamm C, Kanjeekal S, Kay A, et al. Survival of patients with pancreatic cancer treated with varied modalities: A singlecentre study. Mol Clin Oncol. 2017;6(4):583-588. doi: 10.3892/ mco.2017.1179.

6. Briem E, Budkova Z, Sigurdardottir AK, Hilmarsdottir B, Kricker J, Timp W, Magnusson MK, Traustadottir GA, Gudjonsson T. MiR-203a is differentially expressed during branching morphogenesis and EMT in breast progenitor cells and is a repressor of peroxidasin. Mech Dev. 2019;155:34-47. doi: 10.1016/j.mod.2018.11.002.

7. Chen Q-F, Kong J-L, Zou S-C, Gao H, Wang F, Qin SM, Wang W. LncRNA LINC00342 regulated cell growth and metastasis in non-small cell lung cancer via targeting miR-203a-3p. Eur Rev Med Pharmacol Sci. 2019;23(17):7408-7418. doi: 10.26355/ eurrev_201909_18849.

8. Gasparini-Junior J, Fanelli Mf, Abdallah Ea, Chinen Ltd. Evaluating Mmp-2And TgfB-RiExpression InCirculatingTumorCells OfPancreatic Cancer Patients And Their Correlation With Clinical Evolution. ABCD Arq Bras Cir Dig. 2019;32. https://doi.org/10.1590/0102$672020190001 \mathrm{e} 1433$.
9. Gebert LFR, MacRae IJ. Regulation of microRNA function in animals. Nat Rev Mol Cell Biol. 2019;20(1):21-37. doi: 10.1038/ s41580-018-0045-7.

10. Grützmann R. Epidemiology, Treatment, and Outcome of Pancreatic Cancer. Mol. Diagn. Treat. Pancreat. Cancer, Elsevier; 2014, p. 3-9. https://doi.org/10.1016/B978-0-12-408103-1.00001-7.

11. Hou J-M, Krebs MG, Lancashire L, Sloane R, Backen A, Swain RK, Priest LJ, Greystoke A, Zhou C, Morris K, et al. Clinical Significance and Molecular Characteristics of Circulating Tumor Cells and Circulating Tumor Microemboli in Patients With Small-Cell Lung Cancer. J Clin Oncol. 2012;30(5):525-32. doi: 10.1200/ JCO.2010.33.3716

12. Krebs MG, Hou J-M, Sloane $R$, Lancashire L, Priest $L$, Nonaka $D$, Ward TH, Backen A, Clack G, Hughes A, et al. Analysis of Circulating Tumor Cells in Patients with Non-small Cell Lung Cancer Using Epithelial Marker-Dependent and -Independent Approaches. J ThoracOncol.2012;7(2):306-15. doi:10.1097/JTO.0b013e31823c5c16.

13. Livak KJ, Schmittgen TD. Analysis of Relative Gene Expression Data Using Real-Time Quantitative PCR and the 2(-Delta Delta C(T)) Method. Methods.2001;25(4):402-8. doi:10.1006/meth.2001.1262. PMID: 11846609.

14. MaderS, PantelK. Liquid Biopsy:CurrentStatusandFuturePerspectives. Oncol Res Treat. 2017;40(7-8):404-408. doi: 10.1159/000478018.

15. McGuigan A, Kelly P, Turkington RC, Jones C, Coleman HG, McCain RS. Pancreatic cancer: A review of clinical diagnosis, epidemiology, treatment and outcomes. World J Gastroenterol. 2018;24(43):4846-4861. doi: 10.3748/wjg.v24.i43.4846.

16. Murray Np, Reyes E, Tapia P, Badinez L, Orellana N, Fuentealba C, Olivares R, Porcell J, Dueñas R. Redefining micrometastasis in prostate cancer - a comparison of circulating prostate cells, bone marrow disseminated tumorcells and micrometastasis: Implications in determining local or systemic treatment for biochemical failure after radical prostatectomy. Int J Mol Med. 2012;30(4):896-904. doi: 10.3892/ijmm.2012.1071.

17. Panth KM, van den Beucken $T$, Biemans $R$, Lieuwes NG, Weber $M$ Losen $\mathrm{M}$, Yaromina A, Dubois LJ, Lambin P. In vivo optical imaging of MMP2 immuno protein antibody: tumor uptake is associated with MMP2 activity. Sci Rep. 2016;6:22198. doi: 10.1038/srep22198.

18. Poruk KE, Valero V, Saunders T, Blackford AL, Griffin JF, Poling J, Hruban RH, Anders RA, Herman J, Zheng L, et al. Circulating Tumor Cell Phenotype Predicts Recurrence and Survival in Pancreatic Adenocarcinoma. Ann Surg. 2016;264(6):1073-1081. doi:10.1097/ SLA.0000000000001600.

19. Sharma $P$, Alsharif $S$, Fallatah $A$, Chung BM. Intermediate Filaments as Effectors of Cancer Development and Metastasis: A Focus on Keratins, Vimentin, and Nestin. Cells. 2019;8(5):497. doi: 10.3390/ cells8050497.

20. Soldan M. Rastreamento do câncer de pâncreas. Rev Col Bras Cir. 2017;44(2):109-111. English, Portuguese. doi: 10.1590/010069912017002015

21. Wei T, Zhang X, Zhang Q, Yang J, Chen Q, Wang J, Li X, Chen J, Ma T, Li G, et al. Vimentin-positive circulating tumor cells as a biomarker for diagnosis and treatment monitoring in patients with pancreatic cancer. Cancer Lett. 2019;452:237-243. doi: 10.1016/j. canlet.2019.03.009.

22. Winer A, Adams S, Mignatti P. Matrix Metalloproteinase Inhibitors in Cancer Therapy: Turning Past Failures Into Future Successes. Mol Cancer Ther. 2018;17(6):1147-1155. doi: 10.1158/1535-7163. MCT-17-0646.

23. Zhou B, Xu J-W, Cheng Y-G, Gao J-Y, Hu S-Y, Wang L, Zhan HX Early detection of pancreatic cancer: Where are we now and where are we going? Int J Cancer. 2017;141(2):231-241. doi: 10.1002/ ijc.30670.

24. Zhou Y-F, Xu W, Wang X, Sun JS, Xiang JJ, Li ZS, Zhang XF. Negative methylation status of Vimentin predicts improved prognosis in pancreatic carcinoma. World J Gastroenterol.2014;20(36):13172-7. doi: 10.3748/wjg.v20.i36.13172. 